Multimed Tools Appl manuscript No.

(will be inserted by the editor)

\title{
Modeling Immersive Media Experiences by Sensing Impact on Subjects
}

\author{
Eleni Kroupi • Philippe Hanhart • \\ Jong-Seok Lee* . Martin Rerabek . \\ Touradj Ebrahimi
}

Received: date / Accepted: date

\begin{abstract}
As immersive technologies target to provide higher quality of multimedia experiences, it is important to understand the quality of experience (QoE) perceived by users from various multimedia rendering schemes, in order to design and optimize human-centric immersive multimedia systems. In this study, various QoE-related aspects, such as depth perception, sensation of reality, content preference, and perceived quality are being investigated and compared for presentation of $2 \mathrm{D}$ and $3 \mathrm{D}$ contents. Since the advantages of implicit over explicit QoE assessment have become essential, the way these QoE-related aspects influence brain and periphery is also investigated. In particular, two classification schemes using electroencephalography (EEG) and peripheral signals (electrocardiography and respiration) are carried out, to explore if it is possible to automatically recognize the QoE-related aspects under investigation. In addition, a decision-fusion scheme is applied to EEG and peripheral features, to explore the advantage of integrating information from the two modalities. The results reveal that the highest monomodal average informedness is achieved in the high beta EEG band $(0.14 \% \pm 0.09, p<0.01)$, when recognizing sensation of reality. The highest and significantly non-random multimodal average informedness is achieved in when high beta EEG band is fused with peripheral features $(0.17 \% \pm 0.1, p<0.01)$, for the case of sensation of reality. Finally, a temporal analysis is conducted to explore how the EEG correlates for the case of sensation of reality change over time. The

${ }^{*}$ Refers to the corresponding author.

The research leading to these results has been performed in the framework of Swiss National Foundation for Scientific Research (FN 200020-132673-1 and FN 200021-143696-1) and the Basic Science Research Program through the National Research Foundation of Korea funded by the Ministry of Science, ICT and Future Planning, Korea (2013R1A1A1007822).

E. Kroupi, P. Hanhart, M. Rerabek and T. Ebrahimi

Multimedia Signal Processing Group, EPFL, Station 11, CH-1015 Lausanne, Switzerland

E-mail: eleni.kroupi@epfl.ch, philippe.hanhart@epfl.ch, martin.rerabek@epfl.ch, touradj.ebrahimi@epfl.ch

J.-S. Lee

School of Integrated Technology, Yonsei University, Republic of Korea

E-mail: jong-seok.lee@yonsei.ac.kr
\end{abstract}


results reveal that that the right cortex is more involved when sensation of reality is low, and the left one when sensation of reality is high, indicating that approach and withdrawal-related processes occur during sensation of reality.

Keywords EEG · heart rate · respiration · immersiveness · fusion · quality of experience

\section{Introduction}

Most of our impressions and understanding about our surroundings are based on sight. Thus, our perception of the world is mainly three-dimensional. A potential, therefore, actual representation of real scenes should provide a threedimensional feeling to enhance sensation of reality through multimedia devices. The importance of sensation of reality has been recognized in the field of games and virtual reality $[28,35]$, through user-system interactions. Also, recent advances in imaging and displays have enabled implementation of more immersive multimedia environments, offering improved sensation of reality to users [9,20].

As a result, immersive multimedia, which allows users to experience enhanced immersion and involvement in comparison to traditional multimedia, is receiving a rapidly increasing amount of attention. It has strong impact on users' emotion, sense of presence, and degree of engagement, which can eventually be used to provide users more satisfactory media experience $[29,33,34]$. For instance, 3D image and video technologies are gaining ground in multimedia applications since they incorporate depth perception, leading to more realistic scenes, and consequently to emotionally stronger experiences. However, in order for the experience to be as realistic as possible, the quality of the rendering should be as good as possible. Thus, it is important to understand the quality of experience (QoE) perceived by users from various multimedia rendering schemes to design and optimize human-centric immersive multimedia systems.

QoE assessment can be carried out either explicitly or implicitly. In the former case, human subjects are hired and asked to assess their perceived quality of given contents in pre-defined rating scales (e.g., [21]). The analysis and corresponding research outcomes are based on mean opinion scores (MOS) or differential MOS (DMOS) across subjects for various stimuli. However, explicit assessment of QoE can be tiresome and may include subjective biases depending on external factors.

On the other hand, the latter is a bio-inspired approach to automatically recognize the way users perceive and appreciate various multimedia contents, through their physiological signals. Physiological signals can be acquired continuously, realtime, and in a non-invasive way. They originate either from the central nervous system (CNS), such as electroencephalography (EEG), or from the peripheral nervous system (PNS), such as heart rate, respiration, etc. Once an accurate implicit QoE recognition system based on physiological signals is constructed, no explicit response will be required, facilitating real-time monitoring of QoE without subjective biases.

Recently, there have been efforts to measure brain activity in order to understand QoE in 2D and 3D multimedia rendering schemes. In [31], it was demonstrated that abrupt changes in $2 \mathrm{D}$ visual quality give rise to specific components in the EEG, which has potential to be used for implicit subjective quality assessment. 
In the field of 3D image/video, researchers attempted to detect fatigue caused by $3 \mathrm{D}$ visual media based on EEG. In [5], visually evoked potentials in EEG were examined to detect fatigue, where it was shown that the P100 latency (i.e., 100 msec after the stimulus onset) can be used for a fatigue index. The study in [22] showed that the power of the high frequency EEG bands and the changes of the P700 component (i.e., amplitude $700 \mathrm{msec}$ after the stimulus onset) are strong candidates for measuring 3D visual fatigue. Moreover, in [15], it was shown that $3 \mathrm{D}$ visual fatigue is linked to human cortical activities measured by functional Magnetic Resonance Imaging (fMRI). The study in [7] attempted to apply fMRI in combination with magnetoencephalography (MEG) to measure asthenopia and showed potentiality of such a scheme, although detection accuracy remains questionable. These results show that monitoring neurological responses can provide hints for the perceived QoE. However, this topic is still in its infancy with many research questions unanswered. For instance, measuring sensation of reality or depth perception based on EEG and peripheral physiological signals for 3D media has not been adequately considered.

Our previous research attempted to explore these issues. For instance, in [19], a subject-independent classification was performed using EEG and peripheral signals to infer if a subject was experiencing $2 \mathrm{D}$ or $3 \mathrm{D}$ video contents. The results revealed that EEG-based classification can be used to discriminate between $2 \mathrm{D}$ and $3 \mathrm{D}$ contents, independently of the video quality. The EEG high beta frequency band (21-29 Hz) seemed to be mainly responsible for this discrimination. Moreover, in [18] it was demonstrated that EEG-based classification can be also used to automatically recognize high from low sensation of reality, in a subject-dependent framework. Also, classification of sensation of reality from heart and respiration was possible, but less accurate than using EEG signals. Finally, in [17], EEG frontal asymmetry patterns in the EEG alpha band $(8-12 \mathrm{~Hz})$ were observed with respect to perceived quality from $2 \mathrm{D}$ and $3 \mathrm{D}$ stimuli. These patterns indicated right frontal activation when perceived quality was low.

In this paper, we attempt to investigate immersive multimedia presentation experience via explicit subjective rating analysis and implicit monitoring of users' brain and peripheral physiological responses for 2D and 3D multimedia contents. The current study differs from our previous ones in various aspects. Although the database used is the same, in this study subject-independent analysis is conducted, and various QoE-related aspects are investigated and compared. In particular, depth perception, sensation of reality, content preference, and perceived quality are investigated with respect to how they influence each other, and how they influence brain and periphery. More specifically, initially, the subjective ratings are analyzed to investigate how QoE is perceived in an explicit way. Then, two classification schemes using EEG and peripheral signals (ECG and respiration) are carried out, to explore if it is possible to automatically recognize the QoE-related aspects under investigation. In addition, a decision-fusion scheme is applied to EEG and peripheral features, to explore if it is possible to automatically recognize QoE by integrating information from brain and periphery. Finally, the EEG correlates for the case of sensation of reality are investigated over time. For reproducibility reasons, and to encourage further research on the topic, the produced database is 
made publicly available ${ }^{1}$, under the name MIMESIS (Modeling Immersive Media Experiences by Sensing Impact on Subjects).

The paper is organized as follows. Section 2 explains the details of the experiments, the self-assessed ratings and the acquisition of biosignals. In Section 3 the subjective ratings are analyzed and discussed. In Section 4 the feature extraction and classification methods are presented, and the corresponding results are detailed and discussed. Finally, the conclusions are presented in Section 5.

\section{Experiment}

\subsection{Video stimuli}

At the time of this study, the availability of high quality stereoscopic content of sufficient duration to induce immersiveness was almost inexistent. In our experiments, we used video clips recorded during the Montreux Jazz music festival (MJF) by $\mathrm{NVP}^{2} \mathrm{D}^{2}$, a professional 3D video production company. The dataset was composed of eight video contents: one for the training and seven for the tests. All contents were recorded with two RED SCARLET-X cameras ${ }^{3}$ mounted on a Genus Hurricane Rig. All video sequences were recorded in REDCODE RAW (R3D) format ${ }^{4}$, DCI $4 \mathrm{~K}$ resolution $(4096 \times 2160$ pixels $)$, at $25 \mathrm{fps}$, and had a duration of about one minute long. Stereo audio was recorded in PCM format, sampled at $48 \mathrm{kHz}, 24$ bits. Table 1 describes the contents and their characteristics. The recorded video sequences were cropped and downsampled to Full HD resolution $(1920 \times 1080$ pixels $)$ and then compressed with H.264/MPEG-4 AVC. Two different quantization parameters $(\mathrm{QP})$ were selected: $\mathrm{QP}=2$ for high quality (HQ) and $\mathrm{QP}=35$ for low quality (LQ). For each content, four different versions were considered: 2D HQ, 3D HQ, 2D LQ, and 3D LQ, leading to a total of 28 video sequences, 14 of which in $2 \mathrm{D}$ and 14 in $3 \mathrm{D}$.

\subsection{Monitor, sound system and environment}

To display the video stimuli, a HD 46" Hyundai S465D stereoscopic monitor with passive 3D glasses were used. The monitor has a $60 \mathrm{~Hz}$ refresh rate and relies on a line-interleaved display and circular polarizing filters to separate the left- and right-eye images. The laboratory setup was controlled to ensure the reproducibility of results by avoiding involuntary influence of external factors. The test room was equipped with a controlled lighting system with a $6500 \mathrm{~K}$ color temperature and an ambient luminance at $15 \%$ of the maximum screen luminance. For the audio playback, the PSI A14-M professional studio full range speakers ${ }^{5}$ were used.

\footnotetext{
1 http://mmspg.epfl.ch/mimesis

2 http://www.nvp3d.com

3 http://www.red.com/products/scarlet

4 http://www.red.com/learn/red-101/redcode-file-format

5 http://www.psiaudio.com/product/active-monitors/a14-m
} 
Table 1 Characteristics of the contents used in our experiments.

\begin{tabular}{|c|c|}
\hline Content & Description and characteristics \\
\hline Training & $\begin{array}{l}\text { Rock band playing at the Auditorium Stravinski. Dark. Bright spots. } \\
\text { Shot from the back of the auditorium. }\end{array}$ \\
\hline$J a z z$ & $\begin{array}{l}\text { Jazz band playing at the Funky Claude's Lounge at the Opening Party. } \\
\text { Wide shot. }\end{array}$ \\
\hline Rock & $\begin{array}{l}\text { Rock band playing at the Auditorium Stravinski. Dark. Bright spots. } \\
\text { Shot from the back of the auditorium. }\end{array}$ \\
\hline Stage & $\begin{array}{l}\text { MJF general manager on stage introducing the next artist. Very dark. } \\
\text { In French. Wide shot. }\end{array}$ \\
\hline Speech1 & $\begin{array}{l}\text { MJF general manager giving a speech at the Opening Party. In French. } \\
\text { Mid shot. }\end{array}$ \\
\hline Speech2 & Speech at the Opening Party. In French. Mid shot. \\
\hline Outdoor & Crowd walking on the street near the lake. Lot of depth. Wide shot. \\
\hline Interview & Interview of Quincy Jones. Medium close up. \\
\hline
\end{tabular}

\subsection{Participants}

Sixteen subjects ( 5 females, 11 males) took part in our experiments. They were between 19 and 30 years old with an average of 23.8 years of age. All subjects were screened for correct visual acuity, color vision, and stereo vision using the Snellen (no errors on 20/30 line), Ishihara ,and Randot charts, respectively. They all provided written consent forms.

\subsection{Physiological signal acquisition}

The EEG was recorded from 256 electrodes placed at the standard positions on the scalp. An EGI's Geodesic EEG System $300(\mathrm{GES})^{6}$ was used to record, amplify, and digitalize the EEG signals. To ensure that there were more instances than features in the classification schemes (to avoid the curse of dimensionality), only the nineteen electrodes that correspond to the 10-20 International System were used in this study. Additionally, two standard electrocardiogram (ECG) leads were used and placed on the lower left ribcage and on the upper right clavicle, as well as two respiratory inductive plethysmography belts (thoracic and abdomen). All signals were recorded at $250 \mathrm{~Hz}$.

\subsection{Experimental protocol}

Before each experiment, a training session was organized to allow participants to familiarize with the assessment procedure. The content shown in the training session was selected by experts to include $2 \mathrm{D}$ and $3 \mathrm{D}$ examples of various quality levels.

The participants were seated at a distance of 3.2 times the picture height, corresponding to roughly 1.8 meters from the stereoscopic monitor, as suggested in [11]. Experiments were conducted in three sessions. To avoid subjects' fatigue,

\footnotetext{
6 https://www.egi.com/clinical-division/clinical-division-clinical-products/ ges -300
} 
a fifteen-minute break was provided between consecutive sessions. Nine video sequences were presented in the first and second sessions, and ten in the last one, leading to a total of 28 video sequences, and thus, to a total of 28 trials. To reduce contextual effects, the stimuli orders of display were randomized applying different permutation for each subject, whereas the same content was never shown consecutively. The $2 \mathrm{D}$ and $3 \mathrm{D}$ video sequences were mixed, such that subjects could not predict the rendering mode and to reduce any a priori that could influence subjects' ratings and EEG patterns. Therefore, all video sequences were viewed with $3 \mathrm{D}$ glasses. Watching $2 \mathrm{D}$ video content while wearing $3 \mathrm{D}$ glasses reduces the horizontal resolution by a factor two due to characteristics of the monitor used in the experiments (see Section 2.2), which can reduce perceived quality. However, the loss of vertical resolution in passive $3 \mathrm{D}$ display is very low and, in our results, no statistical difference was found between $2 \mathrm{D}$ and $3 \mathrm{D}$ modes on the perceived overall quality (see Section 3).

As illustrated in Figure 1 Each trial consisted of a ten-second baseline period and a stimulus period. The biosignals recorded during the baseline period were used to remove stimulus-unrelated variations from the signals obtained during the stimulus period. During the baseline period, the subjects were instructed to remain calm and focus on a $2 \mathrm{D}$ white cross on a black background presented on the screen in front of them. Once this baseline period was over, a video sequence was randomly selected and presented. After the video sequence was over, the subjects were asked to provide their self-assessed ratings for the particular video sequence without any restriction in time, following the Absolute Category Rating (ACR) evaluation methodology [13].

Once a trial was over, the next baseline period was recorded and the next video sequence was randomly selected, presented and rated. The procedure was repeated until all 28 video sequences were presented and rated.

Regarding the self-assessed ratings, subjects were asked to evaluate the video sequences in terms of four different aspects, namely perceived overall quality, content preference, sensation of reality, and perceived depth quantity. Two different rating scales were used for each aspect, a 9-point and a 3-point scale. The 9-point rating scale ranged from 1 to 9 , with 1 representing the lowest value, and 9 the highest value of each aspect. In particular, the two extremes ( 1 and 9 ) correspond to "low" and "high" for perceived overall quality and content preference, "no presence" and "very strong presence" for sensation of reality, and "no depth" and "a lot of depth" for perceived depth quantity. Regarding the 3-point rating scales, the choices were $\{$ "do not like it", "neutral", "like it" $\}$ for perceived overall quality and content preference, $\{$ "low presence", "middle presence", "high presence" $\}$ for sensation of reality, and \{ "low depth", "middle depth", "high depth" $\}$ for perceived depth quantity. The 3-point scale was intended to be used for classification purposes.

\section{Subjective ratings analysis}

In this section, a subjective ratings analysis is carried out to investigate how QoE is perceived in an explicit way, as well as to explore how various QoE-related aspects are interrelated. 


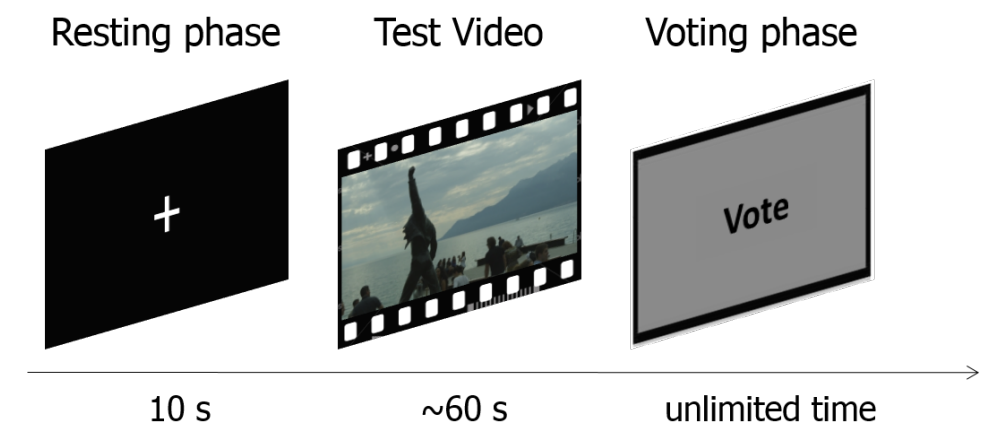

Fig. 1 Example of a trial

Before the analysis on the subjective ratings, outlier detection was performed according to the guidelines described in Section 2.3.1 of Annex 2 of [12], to detect and remove subjects whose ratings appear to deviate significantly from others. During the training session, examples of the lowest and highest quality levels were shown to guide subjects to bound their own perceived overall quality ratings in a similar way. Since quality was the only factor in which subjects could be trained, the outlier detection was performed only on the perceived overall quality ratings. No outliers were detected, thus, for the subjective ratings analysis all sixteen subjects were included.

Regarding the analysis on the subjective ratings, a normality test was performed using the Pearson's chi-squared test to determine if the subjective ratings are well-modeled by a normal distribution. Results showed that, for each individual condition, the ratings of the different subjects were normally distributed. Then, the mean opinion score (MOS) and associated 95\% confidence interval (CI) were computed for each test stimulus, assuming a normal distribution of the subjective ratings, to represent explicit estimates of perceived depth quantity, sensation of reality, content preference and perceived overall quality.

Figure 2 shows the resulting MOS and CI for each case. As it can be observed, for a given quality level, perceived depth and sensation of reality are both higher for 3D when compared to 2D stimuli. Similarly, high quality sequences generally obtained higher ratings for perceived depth quantity, sensation of reality, and perceived overall quality when compared to their corresponding low quality versions. However, the difference in terms of perceived depth and sensation of reality between 3D LQ stimuli and 2D HQ stimuli is not significant as the CIs considerably overlap in all contents. This observation shows that depth cues in $3 \mathrm{D}$ stimuli are effective for depth perception only if a certain level of visual quality is reached. As content Stage is very dark, the perceived 3D effect was not very strong and the perceived depth and sensation of reality were rated relatively low.

To investigate quantitatively whether the objective factors, such as the rendering mode (2D vs. 3D), actual quality level (LQ vs. HQ), and content have a significant influence on the perceptual factors (perceived depth, sensation of reality, content preference and perceived overall quality), an ANOVA analysis was performed on the subjective ratings for each case. In particular, the null hypothesis 


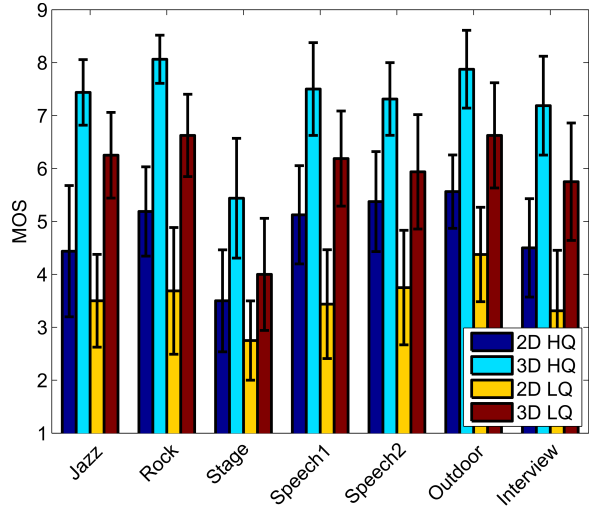

(a) Depth perception

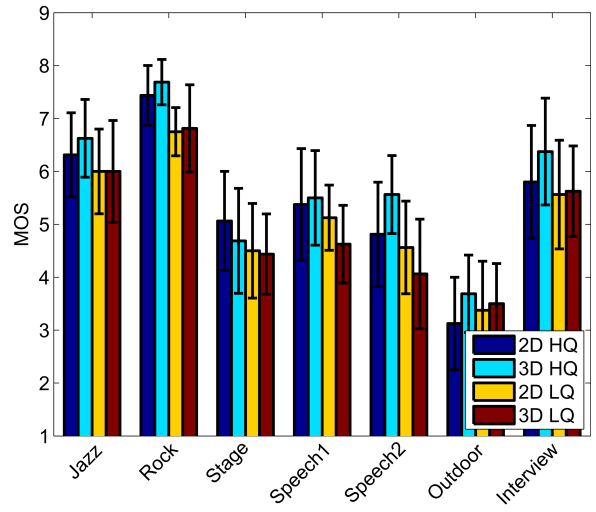

(c) Content preference

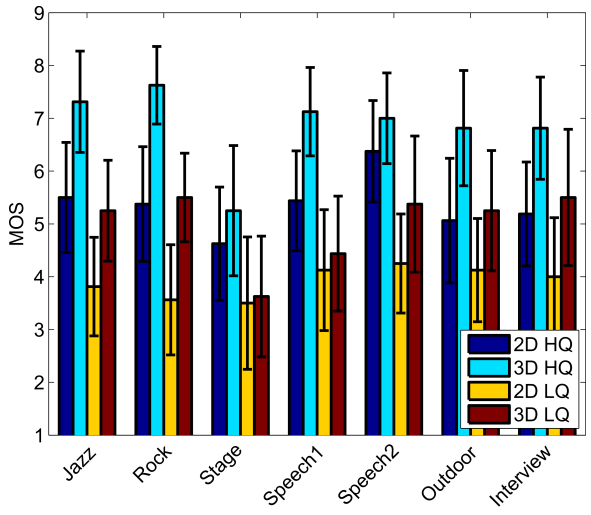

(b) Sensation of reality

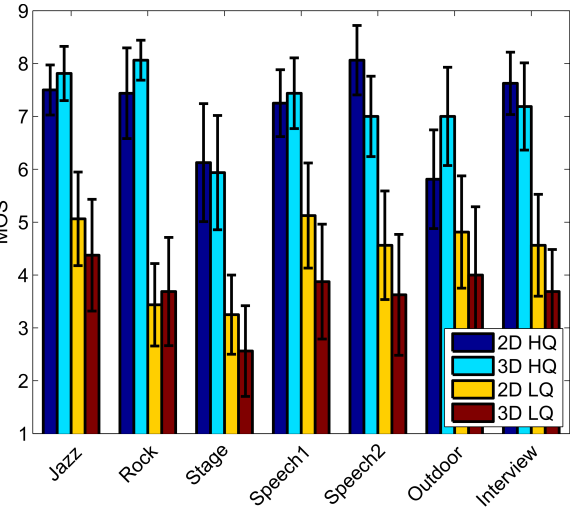

(d) Perceived overall quality

Fig. 2 Mean opinion scores for each of the perceptual factors.

was that the rendering mode, quality level, and content do not influence neither of the perceptual factors.

The null hypothesis was rejected for the cases of perceived depth and sensation of reality for all three objective factors, $p<0.001$, indicating that the effects of the rendering mode, actual quality level, and content on perceived depth quantity and on sensation of reality were significant. Regarding the effects of the objective factors on content preference and on perceived overall quality, only the actual content and the actual quality level influenced these perceptual factors significantly, $p<0.001$. Two sequences (Jazz and Rock) out of seven are from music concert and contain a musical audio track, while the other five sequences are quite general. As the interview of Quincy Jones, who is a famous musician, got similar ratings for content preference when compared to the Jazz sequence, we believe that the presence of a musical audio track was not the only factor influencing content preference. Although the rendering mode itself did not influence neither content preference nor perceived overall quality, the interactions between rendering mode and quality level, as well as the interactions between actual content and quality level influence significantly, $p<0.05$, perceived overall quality. For the rest 
Table 2 Pearson correlation coefficients between the ratings of different perceptual aspects.

\begin{tabular}{|c|c|c|c|}
\cline { 2 - 4 } \multicolumn{1}{c|}{} & $\begin{array}{c}\text { Content } \\
\text { preference }\end{array}$ & $\begin{array}{c}\text { Sensation } \\
\text { of reality }\end{array}$ & $\begin{array}{c}\text { Depth } \\
\text { quantity }\end{array}$ \\
\hline $\begin{array}{c}\text { Overall } \\
\text { quality }\end{array}$ & 0.3392 & 0.7308 & 0.4172 \\
\hline $\begin{array}{c}\text { Content } \\
\text { preference }\end{array}$ & - & 0.3017 & 0.1527 \\
\hline $\begin{array}{c}\text { Sensation } \\
\text { of reality }\end{array}$ & - & - & 0.8835 \\
\hline
\end{tabular}

of the cases, interactions among the objective factors did not influence any other perceptual factor. The findings confirmed our expectations.

To understand the impact of the perceptual factors, such as sensation of reality, content preference, perceived overall quality, and perceived depth quantity on each other, the correlation between the MOS for all four factors was measured using the Pearson correlation coefficient. Table 2 reports the correlation coefficients. The results show that there is a strong correlation between perceived depth quantity and sensation of reality $(\rho>0.88)$. Also, there is a strong correlation between sensation of reality and perceived overall quality $(\rho>0.73)$. However, the correlation between perceived overall quality and perceived depth quantity is relatively low $(\rho=0.42)$, but statistically different from zero, $p=0.03$. Since the correlation between sensation of reality and perceived depth quantity, as well as between sensation of reality and perceived overall quality, is strong, it is rational that the correlation between perceived overall quality and perceived depth quantity is also different from zero, due to the transitivity property. On the other hand, the correlation between perceived depth quantity and content preference $(\rho<0.16)$ is very weak. Thus, apparently content per se impacts on depth perception, but content preference does not. Additionally, depth perception is significantly influenced by the presentation mode, as binocular depth cues are quite powerful, while this factor has no significant effect on content preference, which also explains the weak correlation between content preference and perceived depth. The correlation between sensation of reality and content preference is very low $(\rho<0.3)$ and not statistically different from zero, $p=0.12$. Again, the low correlation between sensation of reality and content preference can be explained by the fact that the rendering mode has a significant impact on first perceptual factor, but not on the former one.

\section{Biosignal analysis}

In this section the acquired biosignals are analysed. In particular, features are extracted from the EEG, ECG, and respiration and then classification is performed to explore if it is possible to discriminate between high and low values of the QoE aspects under investigation. These biosignals were acquired while the subjects were experiencing the audiovisual content. 


\subsection{Feature extraction}

Regarding the EEG signals, the power for frequencies between 4 and $47 \mathrm{~Hz}$ was estimated using Welch periodogram with 128 -sample windows. The mean trial power was then divided by the mean baseline power, in order to extract the power changes without considering the pre-stimulus period. These power changes were captured for different frequency bands, namely the theta $(4-7 \mathrm{~Hz})$, alpha (8-13 $\mathrm{Hz}$ ), beta low $(13-16 \mathrm{~Hz})$, beta middle $(17-20 \mathrm{~Hz})$, beta high $(21-29 \mathrm{~Hz})$, and low gamma $(30-47 \mathrm{~Hz})$ bands.

ECG signals were used to extract the heart rate variability (HRV), which is the physiological measurement of variation in the time intervals between consecutive heart beats, and was estimated using the real-time algorithm developed in [25]. As the HRV is a time-series of nonuniform R-R time intervals (i.e., time intervals between consecutive heart beats), the HRV was regularly resampled at $4 \mathrm{~Hz}$. Respiration signals were low-pass filtered using a second-order Butterworth filter with a cutoff frequency of $1 \mathrm{~Hz}$. Time and frequency-domain features were extracted from HRV and respiration. Regarding the time domain features, the mean, standard deviation, and mean absolute values of the first and second derivatives were extracted from both signals, as in [26]. Regarding the HRV frequency domain features, the power in the low frequency ( $\mathrm{LF}, 0.04-0.15 \mathrm{~Hz}$ ), high frequency $(\mathrm{HF}$, 0.15-0.4 Hz), and the LF/HF ratio were also extracted [1]. Finally, the power in three frequency bands was extracted from respiration, in particular from 0.1-0.2 $\mathrm{Hz}, 0.2-0.3 \mathrm{~Hz}$, and $0.3-0.4 \mathrm{~Hz}$. These features were shown to be related to emotional processes. Thus, in our case, the idea was to explore if the same features can provide information about QoE.

Due to the fact that the duration of the signals was long, EEG spatiotemporal features were used. In particular, EEG power changes in theta, alpha, low beta, middle beta, high beta, and gamma bands were estimated for five second-windows (epochs), leading to twenty-one epochs, and thus, to 399 spatiotemporal features in total. Since peripheral signals need more time to regulate than the EEG signals, only spatial features were extracted from them.

\subsection{Classification scheme}

For the classification schemes, a Linear Discriminant Analysis (LDA) classifier was trained and tested. The LDA classifier was used because it has been shown to increase the accuracy on single-trial EEG analysis $[2,23,31]$. For real-application data, the feature space dimensionality is usually high compared to the number of instances, which leads to a systematic misestimation of the covariance martix [2, 30], and renders classification suboptimal. To overcome this issue, a regularization of the estimated common covariance matrix using shrinkage [30] can be used in the LDA scheme. In particular, the shrinkage parameter is defined as:

$$
\gamma=\frac{d}{(d-1)^{2}} \frac{\sum_{i, j=1}^{n} \operatorname{var}_{\mathrm{k}}\left(\mathrm{z}_{\mathrm{ij}}(\mathrm{k})\right)}{\sum_{i, j=1}^{n} s_{i j}^{2}}
$$


where

$$
z_{i j}(k)=\left(\left(x_{k}\right)_{i}-(\mu)_{i}\right)\left(\left(x_{k}\right)_{j}-(\mu)_{j}\right)
$$

is the correlation coefficient of features $i$ and $j$ of the $k$-th trial ( $x$ represents the feature vector), $\mu$ is the average value across trials, and $d$ is the number of feature vectors in $\mathbb{R}^{n}$ [2]. Also, $s_{i j}$ is the element in the $i$-th row and $j$-th column of the matrix $\Sigma_{c}-\nu I$, where $\Sigma_{c}$ is the empirical covariance matrix, $I$ is the identity matrix, and $\nu$ is the trace $\left(\Sigma_{c}\right) / n$. Therefore, using shrinkage, a better estimate of the covariance matrix is:

$$
\tilde{\Sigma}_{c}=(1-\gamma) \Sigma_{c}+\gamma \nu I .
$$

As performance metric of the classifier, the Informedness was estimated. Informedness is defined as

$$
I=\text { sensitivity }+ \text { specificity }-1,
$$

and is considered an accurate metric of the performance of a classifier with unbalanced classes [27]. Since in our case we deal with unbalanced classes, informedness can be a robust metric for evaluating the classifiers. Sensitivity refers to the true positive rate, and specificity refers to the true negative rate. Obviously informedness takes values in the $[-1,1]$ space, with zero representing the random guess.

Due to the fact that the variance in the performance metrics is reduced in a $K$ fold cross-validation scheme compared to a leave-one-out cross-validation one [8, 36], a two-fold cross-validation was performed on the data, which was repeated ten times to randomize the created folds. The final classification performance metrics were estimated as the average performance metrics across all folds.

\subsection{Classification results}

Regarding the EEG features, classification was performed in the following way; to avoid high dimensional feature spaces, only two temporal features were selected from each training set, based on $I$ (eq. (4)), in a wrapper feature selection scheme. Also, the classification was performed for each frequency band separately, leading to 38 final features per classification scheme (19 electrodes by 2 spatiotemporal features, for six classification schemes). Regarding the ground truth values, the two classes were created based on the 9-point rating scales (the rating values equal to five were excluded as neutral ones, and ratings from one to four corresponded to class 1 , and from six to nine to class 2). The classification was based on the 9-point scales instead of the 3-point ones to have more data for training and testing. The average classification results are presented in Figure 3.

According to Figure 3, the highest average sensitivity $(54.02 \% \pm 13.84)$ and specificity $(60.43 \% \pm 11.52)$ are obtained using the high beta band for predicting sensation of reality. To verify which results are significantly different from a random value, a $t$-test was applied to each case. The null hypothesis was that the sensitivity and specificity for each case follow a Student's $t$ distribution with mean 0.5 (since it is a binary problem, the random value considered was $50 \%$ ). The null hypothesis was rejected with $p<0.01$ for the case of predicting sensation of reality from the high beta band, indicating that the classification result is significantly non-random.

However, typically sensitivity and specificity are not used per se, but are instead combined to form an unbiased metric that takes into account the unbalanced-class 


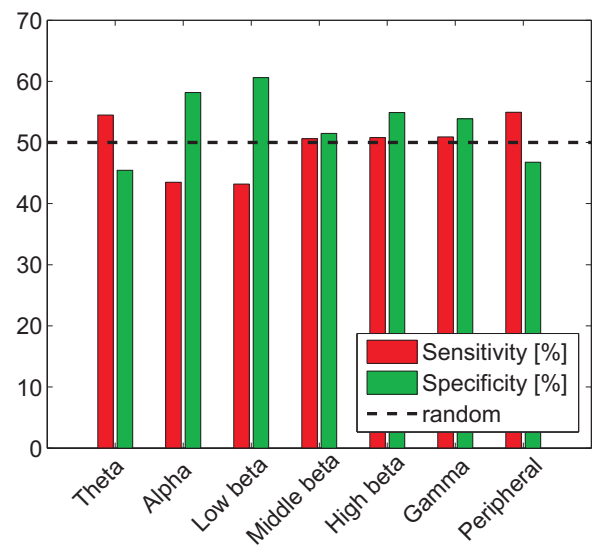

(a) Depth

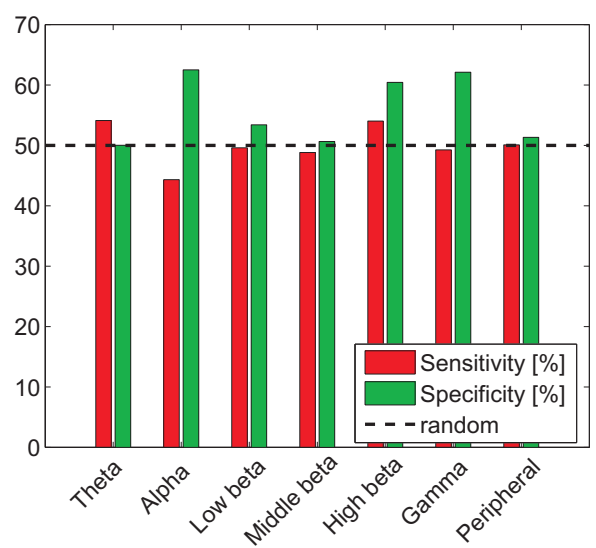

(c) Sensation of reality

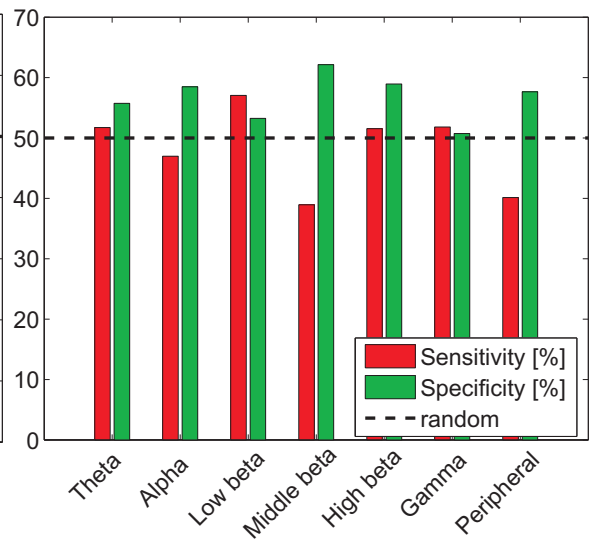

(b) Content Preference

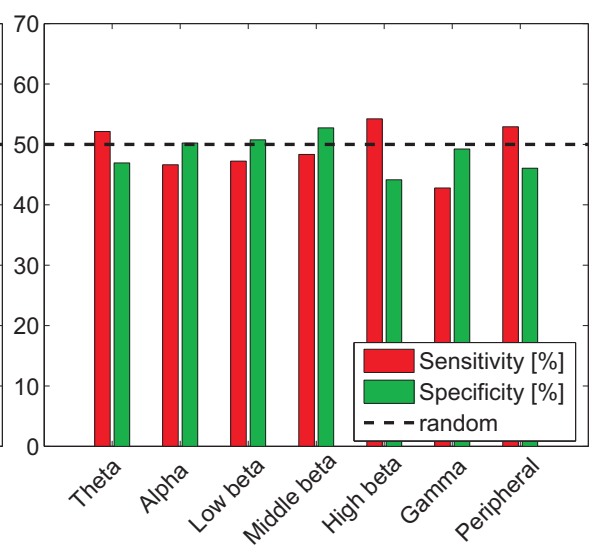

(d) Perceived overall quality

Fig. 3 Sensitivity and specificity for depth perception, content preference, sensation of reality, and overall quality perception. The horizontal line for each case represents the random guess $(50 \%)$.

problem. Thus, to evaluate the performance of the classifiers in an unbiased and a more conventional way, the informedness was estimated (eq. (4)). A $t$-test was applied to the informedness results to estimate whether the values were significantly different from a random value. The results are presented in Figure 4. In consistency with the previous results, the informedness of the high beta band for the case of sensation of reality has the highest value. However, by estimating informedness, three frequency bands are also able to predict depth perception, and four frequency bands are able to predict content preference. Thus, finally, it is indeed possible to predict content preference, depth perception and sensation of reality from EEG signals, but it is not possible to predict them from peripheral signals, and is also not possible to predict overall quality perception under the investigated framework.

These results are also confirmed by integrating all EEG features. In particular, a LDA classifier was trained and tested as previously using all EEG features. The 


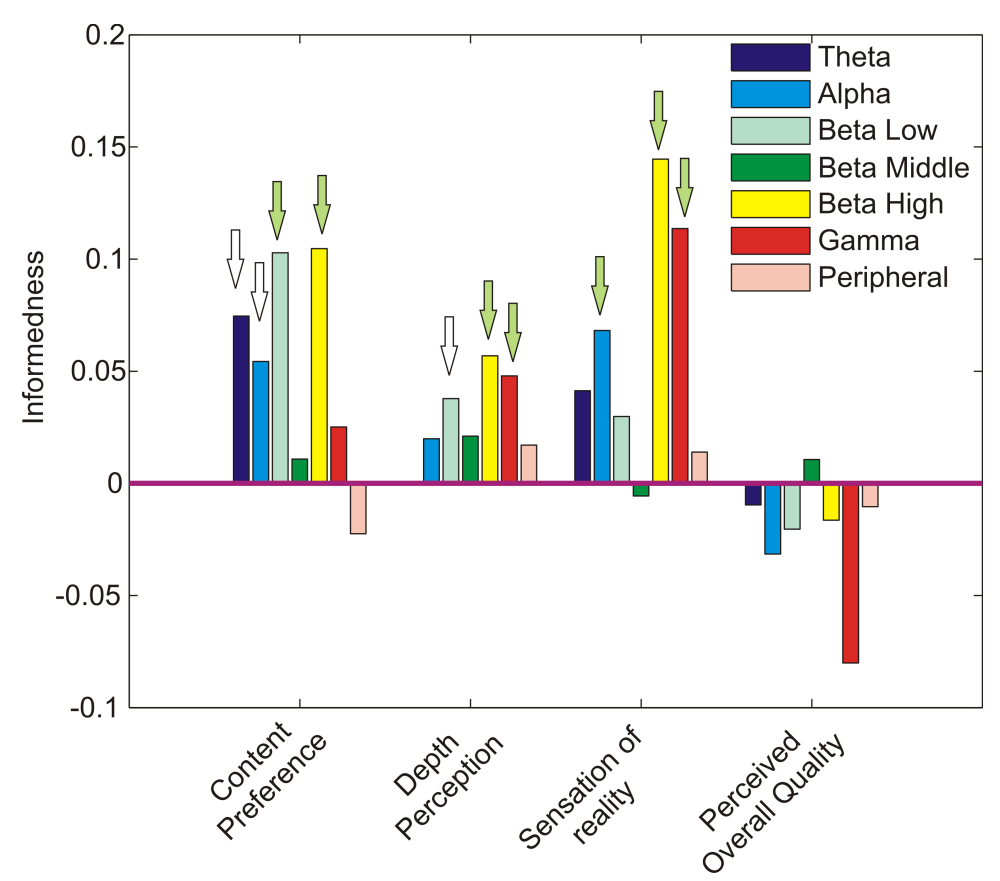

Fig. 4 Informedness for each case. The white arrows point to the frequency bands for which the informedness was significantly different from a random value with $p<0.05$. The light green arrows point to the frequency bands for which the informedness was significantly different from a random value with $p<0.01$. The zero (magenta line) corresponds to the random value.

Table 3 Informedness for all EEG features integrated, for each classification scenario. SD stands for standard deviation. One asterisk indicates significance with $p<0.05$, and two asterisks with $p<0.01$.

\begin{tabular}{|l|c|c|}
\hline Classification tasks & Mean & SD \\
\hline Content Preference** & 0.09 & 0.08 \\
\hline Depth Perception* & 0.04 & 0.09 \\
\hline Sensation of reality** & 0.08 & 0.1 \\
\hline Perceived Overall Quality & -0.03 & 0.07 \\
\hline
\end{tabular}

results are summarized in Table 3 . One may notice indeed that it is possible to predict content preference, depth perception and sensation of reality from EEG signals, as previously. Moreover, in line with Figure 4, the results are better the case of content preference and sensation of reality (i.e., higher mean Informedness and $p<0.01)$. According to Table 3 it is not possible to predict overall quality perception in this context. 


\subsection{Spatial filters}

Since sensation of reality revealed the best results, in this section the EEG correlates for sensation of reality are presented and analysed.

Due to the properties and assumptions on EEG generation [24], it is generally considered that a current source in the brain, $s(t) \in \mathbb{R}^{M \times T_{i}}$, where $M$ is the number of sources and $T_{i}$ is the time, contributes linearly to the scalp potential, $x(t) \in \mathbb{R}^{N \times T_{i}}$, in a way such that

$$
x(t)=A s(t)+n(t),
$$

where $A \in \mathbb{R}^{N \times M}$ is the propagation matrix that represents the strength of contribution of each source to $N$ the surface electrodes. The term $n(t)$ corresponds to the noise, which is not related to the sources. The reverse process of relating the scalp potentials to the sources, is known as backward modeling, and aims at estimating the sources from the scalp potentials. It is formed as

$$
\hat{s}(t)=W^{T} x(t),
$$

where $W$ is either the exact inverse (if it exists) or the pseudoinverse of the matrix $A$. The rows $w^{T}$ of $W^{T}$ are referred to as spatial filters, and can be visualized as scalp maps [2].

A linear classifier trained on spatial features can be considered as a spatial filter [2]. In particular, if $w \in \mathbb{R}^{N}$ is the weight vector, and $x(t) \in \mathbb{R}^{N \times T_{i}}$ represents the EEG signals, then

$$
y(t)=w^{T} x(t)
$$

is the result of spatial filtering. It is known that $w=\Sigma_{c}^{-1}\left(\mu_{2}-\mu_{1}\right)$, where $\Sigma_{c}$ is the estimated common covariance matrix [6]. In this case, $\mu_{2}$ corresponds to low, and $\mu_{1}$ corresponds to high sensation of reality. Thus, a large positive value in a scalp plot indicates activation of a particular part of the cortex when sensation of reality is low, whereas a large negative value in a scalp plot indicates activation of a particular part of the cortex when sensation of reality is high. Moreover, for this case, the common covariance matrix was estimated using shrinkage (eq. (3)). The parameter gamma used to estimate the common covariance matrix was found equal to $\gamma=0.05$ (eq. (1)).

Figure 5 depicts the scalp plots of the high beta band (since it achieved the highest classification performance), for the first sixteen five-second epochs. Similar patterns are observed across all epochs, according to which the right somatosensory cortex is activated when sensation of reality is high, whereas the right parietal cortex is activated when sensation of reality is low. Moreover, particularly in some epochs there is also a slight asymmetry in the frontal cortex. More specifically, the left frontal cortex seems to be activated (in terms of beta band increase) when sensation of reality is low. It is suggested that beta power reflects inhibitory characteristics [14], thus, this finding suggests dominance of the right hemisphere over the left one when sensation of reality is low, due to left hemispheric inhibitory activity. Thus, withdrawal-related processes may occur during low sensation of reality, since this result is consistent with other studies that revealed left frontal beta activation [10,32] or right frontal increase in alpha EEG power [3] during withdrawal-related tasks. This indicates that sensation of reality is related 


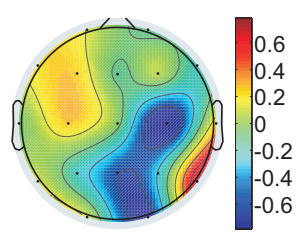

Epoch 1

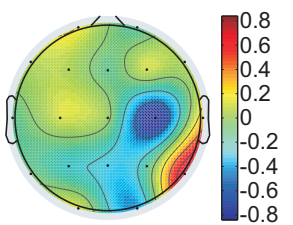

Epoch 5

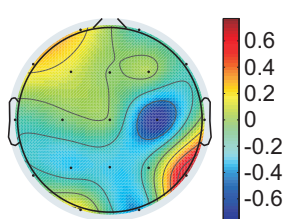

Epoch 9

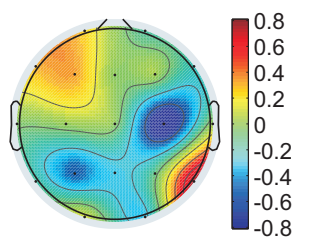

Epoch 13

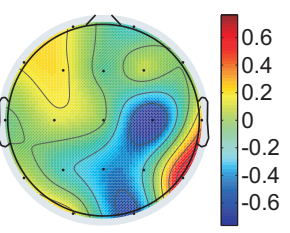

Epoch 2

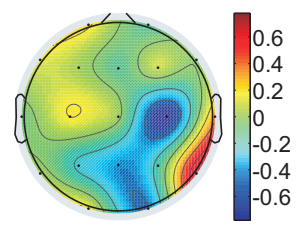

Epoch 6

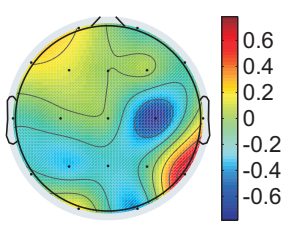

Epoch 10

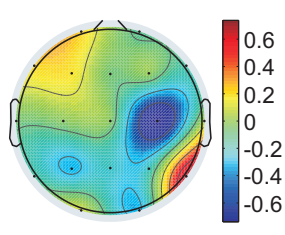

Epoch 14

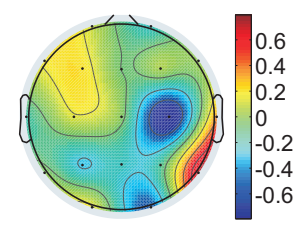

Epoch 3

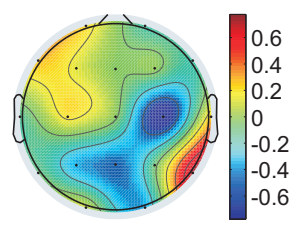

Epoch 7

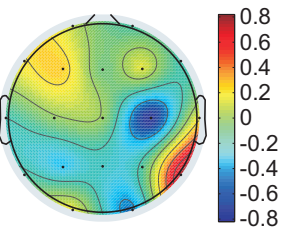

Epoch 11

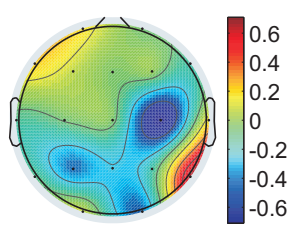

Epoch 15

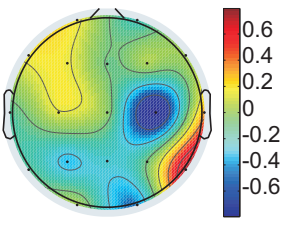

Epoch 4

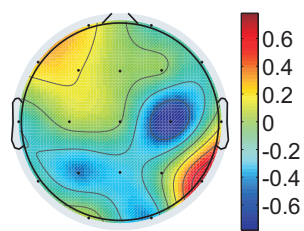

Epoch 8

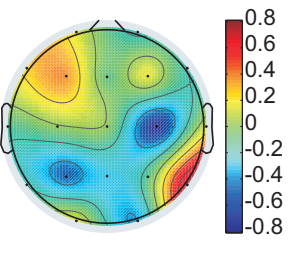

Epoch 12

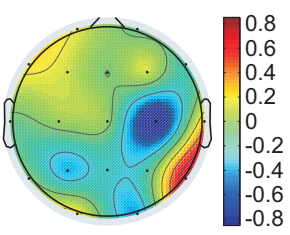

Epoch 16

Fig. 5 Scalp plots that depict the spatial distribution of high beta for each five-second epoch. Scalp plots are presented as top views of the head, the nose is pointing upwards, and the dots indicate the electrode positions. Epochs 12 and 13 provide the highest asymmetry between the right and left frontal cortex, indicating that low sensation of reality is better distinguished from high during these epochs.

to approach/withdrawal-related emotional processes, in the sense that low sensation of reality may lead to withdrawal-related emotional processes, whereas high sensation of reality to approach-related ones.

\subsection{Fusion}

To investigate if it is possible to better recognize QoE by integrating information from both the EEG and the peripheral signals, a decision-fusion scheme based on the maximum probability rule was applied [16]. A decision-fusion scheme 


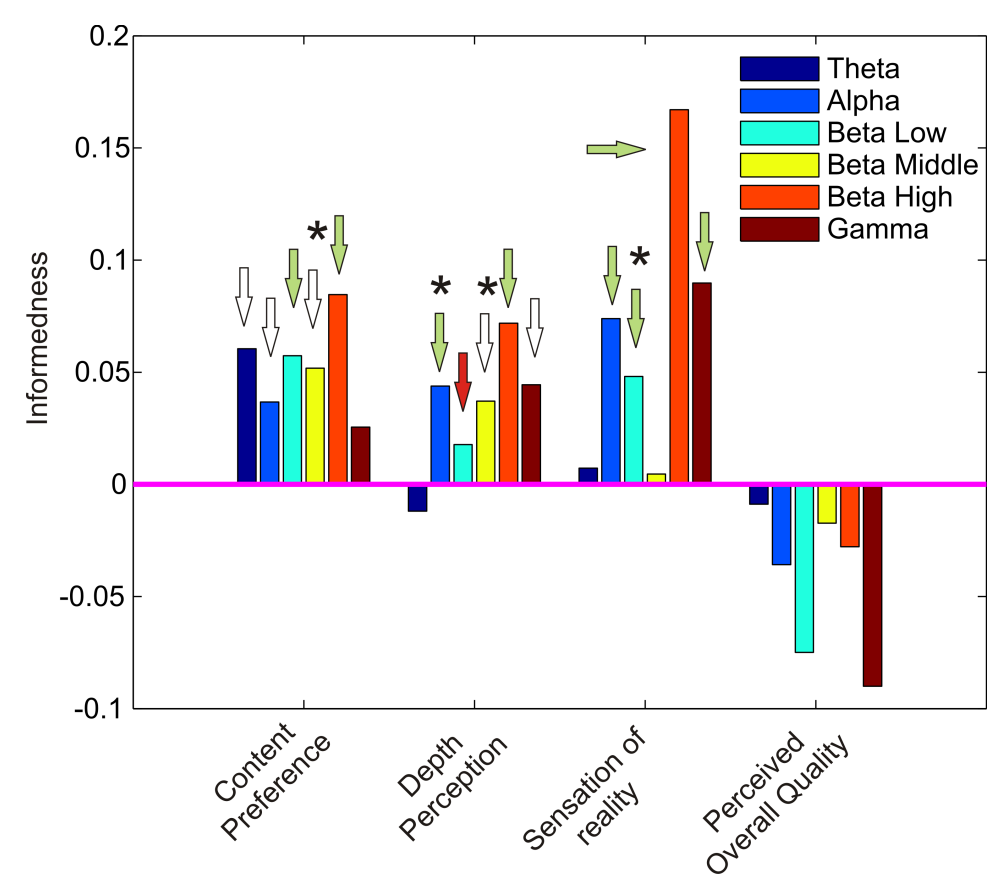

Fig. 6 Informedness for fusion between each frequency band and the peripheral signals. The white arrows point to the frequency bands for which the informedness of fusion was significantly different from a random value with $p<0.05$. The light green arrows point to the frequency bands for which the informedness of fusion was significantly different from a random value with $p<0.01$. The zero (magenta line) corresponds to the random value. The asterisks correspond to the frequency bands for which the informedness was significantly different from a random value only when these frequency bands were fused with peripheral signals, and not when they were used alone. The red arrow shows the only case which was significantly different from random guess without fusion whereas it was not with fusion.

is usually preferred from a feature-fusion one, in order to avoid the curse of dimensionality that may be present due to an increase in the number of features. Since there are only two modalities involved (EEG and peripheral features in each case), the final decision for the assigned class was based on the classifier with the highest posterior probability. The classifiers were equally weighted, which implies that the classifiers were considered equally important.

The results are presented in Figure 6. To compare the multimodal fusion results with the results obtained using single modalities (i.e., EEG or peripheral signals), a $t$-test was applied to each case (e.g., theta band alone or theta band fused with peripheral signals). The cases in which the informedness was significantly higher with fusion compared to only using EEG are marked with asterisks in Figure 6. Thus, one can observe that fusing peripheral features with EEG features leads to slightly better results than only using EEG or peripheral features. In particular, four more EEG frequency bands lead to significantly better than random results when fused with peripheral signals, two of which with $p<0.01$. Only the low beta band led to significantly better informedness for the depth perception case when not fused with the peripheral signals. Finally, the average percentage of the 
contribution of the two classifiers was estimated to investigate whether one of the two classifiers (EEG-based or periphery-based classifiers) contributed more than the other. As expected, the decision was based on the EEG classifiers for $64 \%$ of the cases, and for $36 \%$ of the cases it was based on the peripheral classifiers.

\subsection{Further discussion}

In our first study [19], a subject-independent classification was performed to infer if a subject was experiencing $2 \mathrm{D}$ or $3 \mathrm{D}$ video contents. In [18], we demonstrated that classification can be also used to automatically recognize high from low sensation of reality, in a subject-dependent framework. In this paper, we aimed at predicting all evaluated aspects, i.e., content preference, depth perception, sensation of reality, and perceived overall quality, using subject-independent classification systems. The current study also differs in the sense that we used an LDA classifier, whereas a SVM classifier was used in $[18,19]$. Moreover, in this paper, we also applied a decision-fusion scheme to EEG and peripheral features, to explore the advantage of integrating information from the two modalities. Finally, in this paper, we investigated the EEG correlates over time for the case of sensation of reality, whereas EEG correlates for the case of overall perceived quality were investigated without considering the time evolution in [17].

In this study we demonstrated that the high beta EEG band is mainly responsible for discriminating high from low sensation of reality. This finding corroborates with the finding in [19], in which it was shown that high beta EEG band is mainly responsible for discriminating $2 \mathrm{D}$ from $3 \mathrm{D}$ video contents. Thus, apparently similar brain patterns occur during experience of $2 \mathrm{D}$ and $3 \mathrm{D}$ contents, as well as during sensation-of-reality experiences, indicating that these two processes may actually be related, which is in line with our expectations. This observation is also supported by [4], in which it was shown that stronger emotions are elicited with $3 \mathrm{D}$ compared to $2 \mathrm{D}$ visual stimuli, reflected in the activity of the right amygdala. Since sensation of reality typically provokes stronger emotions due to its resemblance with the real world, in [4] it is expected that sensation of reality was higher with $3 \mathrm{D}$ compared to $2 \mathrm{D}$ stimuli, although the participants in the experiments were not explicitly asked about that. In general EEG provides a very good time resolution and fMRI spatial resolution. Thus, it is expected that fusion of both brain imaging tools will significantly improve classification performance.

Moreover, we found that frontal-cortex asymmetry patterns occur during sensation of reality, indicating that approach-related and withdrawal-related processes may take place during such experiences. In [17] it was shown that frontal asymmetry patterns also occur with perceived quality. In particular, the right frontal cortex is activated when perceived quality is low, indicating that withdrawal-related processes are involved in such experiences. Since in this study we have found that the right frontal cortex is activated when sensation of reality is low, this finding indicates that similar brain patterns occur during perceived quality and sensation of reality. This corroborates also with the subjective rating analysis, in which it was shown that sensation of reality and perceived overall quality are correlated. However, although the classification performance for sensation of reality was significantly non-random, this was not the case for overall perceived quality. This may be due to occurrence of weaker brain patterns during overall quality compared to 
the patterns provoked by sensation of reality, which may affect the classification performance. More specifically, sensation of reality may be correlated with overall perceived quality, but it is also correlated with additional factors (e.g., 2D or 3D rendering), which may lead to more distinguishable EEG patterns. Moreover, another possible reason for not being able to recognize overall quality perception may be due to subjective interpretation of the question. In particular, overall quality is a mix of different factors, such as picture quality, depth quality, etc. and each subject may weight those factors differently and come up with his/her own definition of overall quality based on what mainly matters to him/her.

\section{Conclusions}

In this study the way various QoE-related aspects affect brain and periphery was explored. In particular, an experiment with sixteen participants was conducted, during which the participants were experiencing 2D and 3D multimedia contents of various quality levels, while at the same time their EEG, ECG, and respiration signals were recorded. The subjects provided their self-assessed ratings after each video, in which they were asked to rate various aspects that may influence QoE, namely, perceived depth, perceived overall quality, content preference, and sensation of reality. A subjective ratings analysis revealed that the effects of the rendering mode, actual quality level, and content on perceived depth and on sensation of reality were significant. It also revealed that there is a strong correlation between perceived depth and sensation of reality, as well as between sensation of reality and perceived overall quality. Finally, for a given quality level perceived depth and sensation of reality are both higher for 3D when compared to 2D stimuli. Similarly, high quality sequences generally obtained higher ratings for perceived depth quantity, sensation of reality, and perceived overall quality when compared to their corresponding low quality versions. However, the difference in terms of perceived depth and sensation of reality between 3D LQ stimuli and 2D HQ stimuli was not significant.

To investigate if it is possible to automatically recognize perceived depth, sensation of reality, content preference, and perceived overall quality, two classification schemes were carried out, one using EEG and another peripheral features. It was revealed that it is possible to recognize perceived depth, content preference, and sensation of reality from EEG signals, but not from the peripheral ones. It was also found that EEG high beta band is the main responsible one for each case, and that the left frontal cortex seems to be involved when sensation of reality is high, indicating that high sensation of reality is related to approach-related emotional processes. Finally, decision fusion between peripheral and EEG features was found to improve classification performance in some cases.

\section{References}

1. Bilchick, K.C., Berger, R.D.: Heart rate variability. Journal of Cardiovascular Electrophysiology 17(6), 691-694 (2006)

2. Blankertz, B., Lemm, S., Treder, M., Haufe, S., Müller, K.R.: Single-trial analysis and classification of ERP components: a tutorial. NeuroImage 56(2), 814-825 (2011) 
3. Davidson, R., Ekman, P., Saron, C., Senulis, J., Friesen, W.: Approach-withdrawal and cerebral asymmetry: Emotional expression and brain physiology: I. Journal of Personality and Social Psychology 58(2), 330-341 (1990)

4. Dores, A.R., Barbosa, F., Monteiro, L., Leitão, M., Reis, M., Coelho, C.M., Ribeiro, E., Carvalho, I.P., Sousa, L., Castro-Caldas, A.: Amygdala activation in response to 2D and 3D emotion-inducing stimuli. PsychNology Journal 12(1-2), 29-43 (2014)

5. Emoto, M., Niida, T., Okana, F.: Repeated vergence adaptation causes the decline of visual functions in watching stereoscopic television. Journal of Display Technology 1(2), 328-340 (2005)

6. Fukunaga, K.: Introduction to Statistical Pattern Recognition. Academic press (1990)

7. Hagura, H., Nakajima, M., Owaki, T., Takeda, T.: Study of asthenopia caused by the viewing of stereoscopic images: measuring by MEG and other devices. In: Proceedings of SPIE, vol. 6057, pp. 192-202 (2006)

8. Hastie, T., Tibshirani, R., Friedman, J.: The Elements of Statistical Learning, vol. 2. Springer (2009)

9. Hayward, V., Astley, O.R., Cruz-Hernandez, M., Grant, D., Robles-De-La-Torre, G.: Haptic interfaces and devices. Sensor Review 24(1), 16-29 (2004)

10. Hofman, D., Schutter, D.J.: Asymmetrical frontal resting-state beta oscillations predict trait aggressive tendencies and behavioral inhibition. Social Cognitive and Affective Neuroscience pp. 1-8 (2011)

11. ITU-R BT.2021: Subjective methods for the assessment of stereoscopic 3DTV systems. International Telecommunication Union (2012)

12. ITU-R BT.500-13: Methodology for the subjective assessment of the quality of television pictures. International Telecommunication Union (2012)

13. ITU-T P.910: Subjective video quality assessment methods for multimedia applications. International Telecommunication Union (2008)

14. Jensen, O., Goel, P., Kopell, N., Pohja, M., Hari, R., Ermentrout, B.: On the human sensorimotor-cortex beta rhythm: sources and modeling. Neuroimage 26(2), 347-355 (2005)

15. Kim, D., Jung, Y.J., Kim, E., Ro, Y.M., Park, H.W.: Human brain response to visual fatigue caused by stereoscopic depth perception. In: Proc. Int. Conf. Digital Signal Processing, pp. 1-5. Corfu, Greece (2011)

16. Kittler, J., Hatef, M., Duin, R.P., Matas, J.: On combining classifiers. IEEE Transactions on Pattern Analysis and Machine Intelligence 20(3), 226-239 (1998)

17. Kroupi, E., Hanhart, P., Lee, J.S., Rerabek, M., Ebrahimi, T.: EEG correlates during video quality perception. In: Proceedings of the 22nd European Signal Processing Conference (EUSIPCO), Lisbon, Portugal (2014)

18. Kroupi, E., Hanhart, P., Lee, J.S., Rerabek, M., Ebrahimi, T.: Predicting subjective sensation of reality during multimedia consumption based on EEG and peripheral physiological signals. In: Proceedings of the IEEE International Conference on Multimedia and Expo (2014)

19. Kroupi, E., Hanhart, P., Lee, J.S., Rerabek, M., Ebrahimi, T.: User-independent classification of $2 \mathrm{D}$ versus $3 \mathrm{D}$ multimedia experiences through EEG and physiological signals. In: Proceedings of the 8th International Workshop on Video Processing and Quality Metrics for Consumer Electronics-VPQM (2014)

20. Kulkarni, S.D., Minor, M.A., Deaver, M.W., Pardyjak, E.R., Hollerbach, J.M.: Design, sensing, and control of a scaled wind tunnel for atmospheric display. IEEE/ASME Transactions on Mechatronics 17(4), 635-645 (2012)

21. Lee, J.S., De Simone, F., Ebrahimi, T.: Subjective quality evaluation of foveated video coding using audio-visual focus of attention. IEEE Journal of Selected Topics in Signal Processing 5(7), 1322-1331 (2011)

22. Li, H.C.O., Seo, J., Kham, K., Lee, S.: Measurement of 3D visual fatigue using eventrelated potential (ERP): 3D oddball paradigm. In: Proc. 3DTV Conf., pp. 213-216. Istanbul, Turkey (2008)

23. Muller, K., Anderson, C.W., Birch, G.E.: Linear and nonlinear methods for brain-computer interfaces. IEEE Transactions on Neural Systems and Rehabilitation Engineering 11(2), 165-169 (2003)

24. Nunez, P., Srinivasan, R.: Electric Fields of the Brain: The Neurophysics of EEG. Oxford University Press (2006)

25. Pan, J., Tompkins, W.J.: A real-time QRS detection algorithm. IEEE Trans. Biomedical Engineering (3), 230-236 (1985) 
26. Picard, R., Vyzas, E., Healey, J.: Toward machine emotional intelligence: Analysis of affective physiological state. Pattern Analysis and Machine Intelligence, IEEE Transactions on 23(10), 1175-1191 (2001)

27. Powers, D.M.W.: Evaluation: From precision, recall and f-measure to ROC, informedness, markedness \& correlation. Journal of Machine Learning Technologies 2(1), 37-63 (2011)

28. von der Pütten, A.M., Klatt, J., Broeke, S.T., McCall, R., Krämer, N.C., Wetzel, R., Blum, L., Oppermann, L., Klatt, J.: Subjective and behavioral presence measurement and interactivity in the collaborative augmented reality game TimeWarp. Interacting with Computers 24, 317-325 (2012)

29. Sanchez-Vives, M.V., Slater, M.: From presence to consciousness through virtual reality. Nature Reviews Neuroscience 6(4), 332-339 (2005)

30. Schäfer, J., Strimmer, K.: A shrinkage approach to large-scale covariance matrix estimation and implications for functional genomics. Statistical Applications in Genetics and Molecular Biology 4(1), 32 (2005)

31. Scholler, S., Bosse, S., Treder, M.S., Blankertz, B., Curio, G., Müller, K.R., Wiegand, T.: Toward a direct measure of video quality perception using EEG. IEEE Transactions on Image Processessing 21(5), 2619-2629 (2012)

32. Schutter, D.J., de Weijer, A.D., Meuwese, J.D., Morgan, B., Van Honk, J.: Interrelations between motivational stance, cortical excitability, and the frontal electroencephalogram asymmetry of emotion: a transcranial magnetic stimulation study. Human Brain Mapping $\mathbf{2 9}(5), 574-580$ (2008)

33. Slater, M.: Place illusion and plausibility can lead to realistic behaviour in immersive virtual environments. Philosophical Transactions of the Royal Society B: Biological Sciences 364(1535), 3549-3557 (2009)

34. Slater, M., Wilbur, S.: A framework for immersive virtual environments (FIVE): Speculations on the role of presence in virtual environments. Presence: Teleoperators and Virtual Environments 6(6), 603-616 (1997)

35. Stoakley, R., Conway, M.J., Pausch, R.: Virtual reality on a WIM: interactive worlds in miniature. In: Proc. SIGCHI Conf. Human Factors in Computing Systems, pp. 265-272. Denver, CO (1995)

36. Theodoridis, S., Koutroumbas, K.: Pattern Recognition. Academic Press (1998) 